\title{
Supplement to the Theory of Relativity
}

\author{
Xiaoliang Miao"
}

Chongqing Branch of Shanghai Pacific Pump Group Co., Ltd. Jiangbei District, Chongqing, China; 708981976@ qq.com

Abstract: The problem about "who is right in relativity and Newtonian mechanics" is analyzed and discussed, and the theoretical results described in this study are only used as reference. This study reveals that there is no contradiction between relativity and Newtonian mechanics, and the essence of the relativity lies in the mass energy equation.

Keywords: Theory of Relativity; Newtonian Mechanics

The differences between the concept of theory of relativity and Newtonian mechanics are explained, thereby it allows to understand the difference and connection between the two concepts. The first concluded difference of the basic operation modes of earth and the planets in theory of relativity and Newtonian mechanism is shown as Figure 1 firstly.

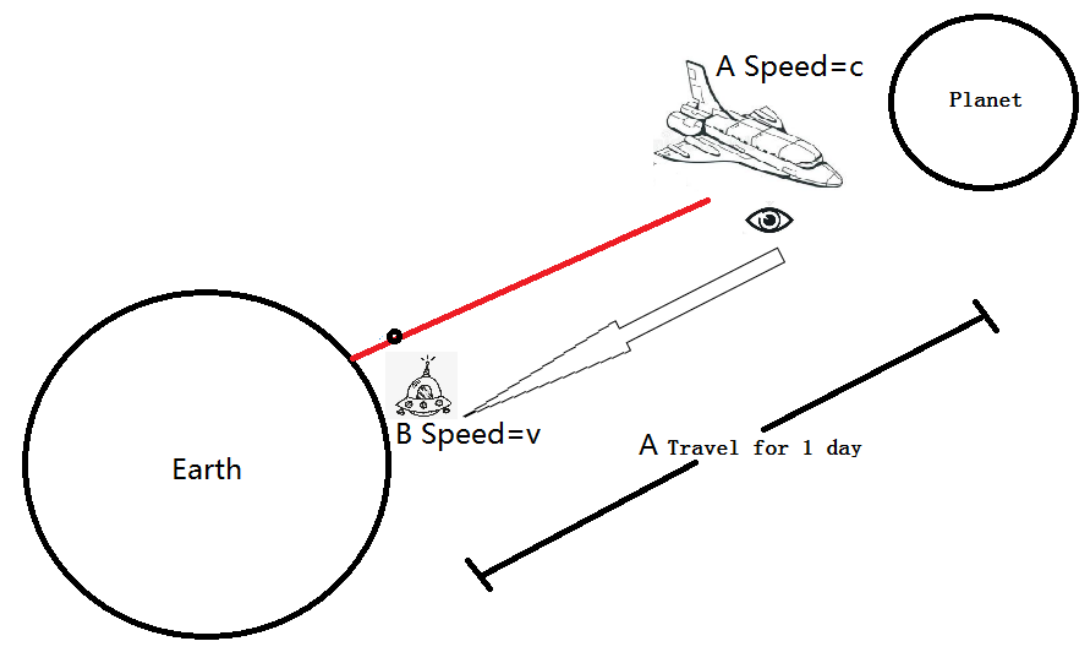

Figure 1. Basic operation modes of earth and planets.

The following two to-be-discussed questions are concluded by the preliminary discussions. Firstly, if a light spacecraft $A$ is launched from the earth to a planet, which is provided with an infinitely long chain, a monkey spacecraft follows the light spacecraft A with the chain as the orbit at the earth normal speed v. Both spacecrafts are launched at the same time. A day is required for the light spacecraft A driven to the planet, and the chain behind the spacecraft has the length $\mathrm{L}$ after the planet is reached ${ }^{[1]}$.

Question 1: where is the monkey spacecraft when the light spacecraft is driven to the planet?

Question 2: what would you see if you look at the monkey spacecraft through binoculars during the flight of the light spacecraft?

If the above two questions should be answered accurately, the knowledge of relativity is required firstly, and the

This is an open-access article distributed under the terms of the Creative Commons Attribution Non-Commercial License (http://creativecommons. org/licenses/by-nc/4.0/), which permits unrestricted non-commercial use, distribution, and reproduction in any medium, provided the original work is properly cited. 
existing time delay effect is combined for analysis. The details are shown in Figure 2.

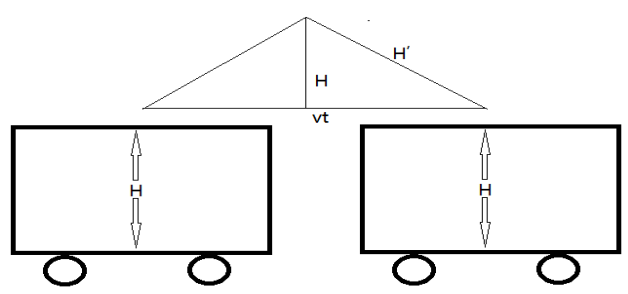

Figure 2. Conceptual diagram of time delay effect.

Figure 2 shows that a light pulse is sent vertically up from the floor of the car if a person is in the car, which is then reflected back to the bottom of the car, according to the scenario simulation concept after the above two problems are solved. The time for the observer in the car to see the light is recorded as t0, the height of the car is $\mathrm{H}$, and the speed of light is $\mathrm{c}$, then

\section{$2 \mathrm{H}=\mathrm{c}^{*} \mathrm{t}_{0}(1)$}

Then, the time for the observer to move outside the car for seeing the light is recorded as $t$, then

$2 \mathrm{H}^{\prime}=\mathrm{c} * \mathrm{t}(2)$

Meanwhile, the car will continuously travel at the speed $\mathrm{v}$ and travel time $\mathrm{t}$, then the trigonometric function relationship can be obtained, $H^{\prime 2}=H^{2}+\left(\frac{1}{2} v t\right)^{2}(3)$

Then, it is put into the functional relation to obtain the follows:

$$
t=\frac{t_{0}}{\sqrt{1-\left(\frac{v}{c}\right)^{2}}}
$$

The following formula is set as the important node:

$\frac{v}{c}=\beta$

The follows is obtained finally:

$$
t=\frac{t_{0}}{\sqrt{1-\beta^{2}}}
$$

The derivation of the time delay effect in relativity is shown as the above contents, and the same scale formula is also derived: $l=l_{0} \sqrt{1-\beta^{2}}$

In the above equation, $l$ refers to the object in movement, and $l_{0}$ refers to the object before movement ${ }^{[2]}$.

Therefore, the example in the relativity shows that the light movement direction is different from that of the car, as they move in a plane, then the trigonometric function should be used. Some change is made so that the light is moving in the same direction as the car. They move in a straight line as Figure 3.

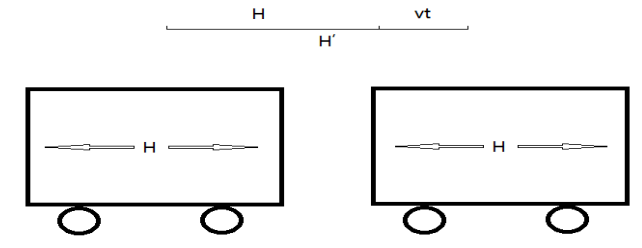

Figure3. Schematic diagram of the movement direction of light and car in trigonometric function.

The follows can be obtained through observation in the above figure:

$H^{\prime}=H+\operatorname{vt}(8)$

Therefore, the distance of light movement is that of the light movement in the car plus that of car movement when the observer is outside the $\operatorname{car}^{[3]}$. Then, it is applied into the above formula, and the follows can be obtained:

$t=\frac{t_{0}}{1-\beta}(9)$

It looks simpler. The conclusion is similar to the result of movement on the plane. The $\beta$ denominator is larger, 
and the error is smaller. The above formula shows that: $t-t_{0}=\Delta t=\beta t$, time is expanded, the scale formula should also be $l-l_{0}=\Delta l=-\beta l$, the object is shortened, and the value of expansion and shortening is the product of the coefficient $\beta$. However, the Einstein formula is not complete, and the object time and space are changed in the movement. When the object is stopped, time and space are changed back to the original value. The teacher's formula only shows the change process rather than the return process, thereby causing the contradiction with Newtonian mechanics. Einstein's data is more accurate in the real world, while Newton is more reasonable. So far, proponents of the two schools of scholarship have argued continuously. It is proposed in relative studies that Newton works at low speeds and relativity works at high speeds in order to resolve the contradiction. However, the data obtained from the relativity concept is more accurate. It is also the fact in the study. There must be errors in all object movements regardless of high speed or low speed. The current GPS navigation is the research result after relativity correction.

Then, the existing discussion data shows that the contradiction is deduced. In fact, both parties are not wrong, while mutual theories are correct to certain degree. The most important $\beta$ is shown in Figure 4.

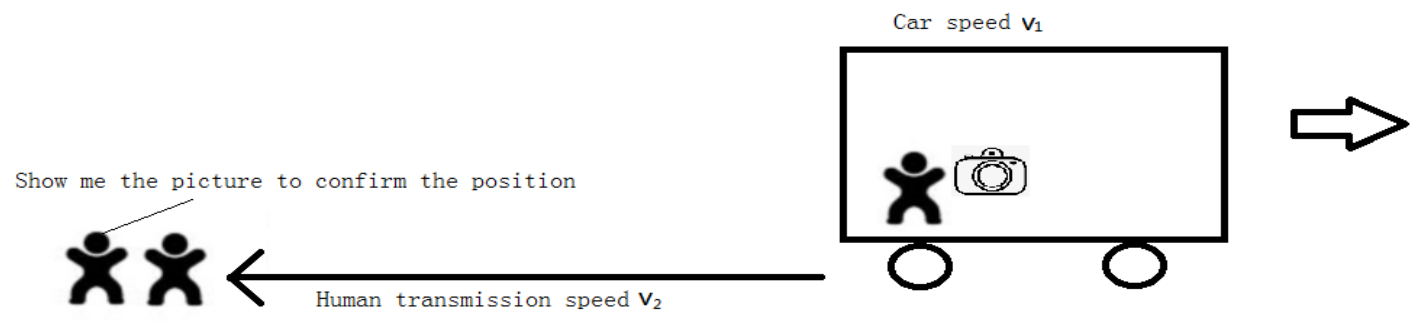

Figure 4. Schematic diagram of the speed of the man and the car.

Figure 4 shows that if a car starts to the front of an observer at speed v1, a transmitter will come down from the car after a fixed period of time ${ }^{[4]}$. A position picture of the car is shot and transmitted to the observer at the initial point at the speed v2. Is the picture observed by the observer the instant position of the car? Obviously not, what is the error? v1 $=50 \mathrm{~m} / \mathrm{s}, \mathrm{v} 2=100 \mathrm{~m} / \mathrm{s}$, the observer distance is $100 \mathrm{~m}$, the instant transmitter shoots the picture to the observer, $\frac{S}{v_{2}}=1 \mathrm{~s}$ , namely the picture is transmitted to the observer within 1 second. The car is moved continuously ${ }^{[5]}$. The transmitter comes down and moves forwards continuously. The transmitter arrives at the observer, who drives continuously for $1 \mathrm{~s}^{*} \mathrm{v} 1=50 \mathrm{~m}$. The distance to the observer is $\mathrm{S}^{\prime}=150 \mathrm{~m}$, the error is 1 second. The position for the observer to see the picture is $\mathrm{S}=100 \mathrm{~m}$. The error is $50 \mathrm{~m}$, namely $\triangle \mathrm{S}=50 \mathrm{~m}$. The position of 1 second *v2 is observed, it is actually the position of 1.5 second *v2. Then, when the car is driven for 2 seconds, another transmitter comes down for the same operation, the time required by the transmitter is $2 \mathrm{~s}$, the error is $\triangle \mathrm{S}=100 \mathrm{~m}$, the car is on the position of $300 \mathrm{~m}$, the observer only obtains the picture of $200 \mathrm{~m}$. The position of 2 seconds * 2 is observed, and the man gets on the car at the

position of 3 seconds $* \mathrm{v} 2 . \triangle \mathrm{t}=1.0 \mathrm{~s}$. The error should be $\Delta \mathrm{t}=\frac{v_{1}}{v_{2}} t$. Namely as long as $v_{1}$ is not 0 , the error value always exists, the error value is related to the value $v_{2}$. If $v_{2}$ is larger, the error value is smaller. Meanwhile, because the transmitter speed and interval are not changed, the distance is larger and larger, the observed picture speed is also slower and slower ${ }^{[6]}$.

Then, the speed $v_{2}$ of the transmitter is converted to the light speed c. Light is the same as the transmitter in daily life. The object is transmitted to the eyes through pictures frame by frame. Even the light is faster, the slower object relative to the observer must produce errors in front of the object, the formula is $\frac{v_{1}}{c}$, it is just the coefficient $\beta$, and then it can be called light error coefficient.

The movement in Newtonian mechanics refers to the ontology movement of the object back to the question of relativity ${ }^{[7]}$. The movement in theory of relativity refers to the observation to object movement. It refers to the information received by the observer. Teacher Einstein is lack of detailed description of the relationship to the observer position. Is the speed of the observer to observe the object at each position the same? Are the speed and direction of 
observing the object the same?

The speed is a scalar if the observer is inside the object, and the speed is a vector if the observer is outside the object. If a person is in a car, who cannot see the external reference, he has no idea about the direction that the object is moving in. If the observer is outside, it is easy for the observer to know the direction that the object is moving in even without the reference, then the speed of the object becomes a vector with the observer as the reference.

In short, there are three observers B, H, A. They jointly observe the movement of the train by standing in front, middle and back of the train respectively as shown in Figure 5.

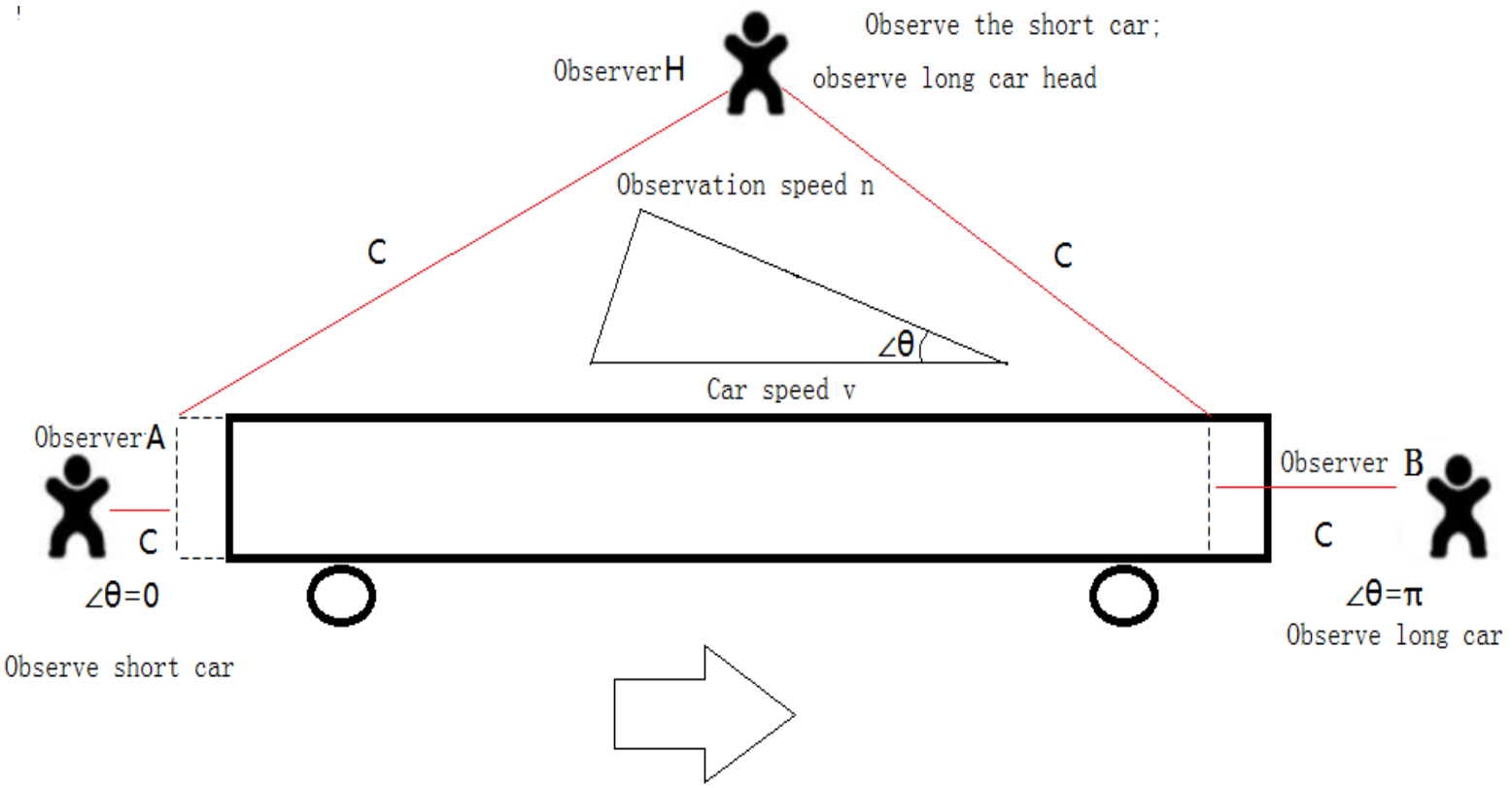

Figure 5. Movement trajectory figure of different observers and the train.

The above figure shows that the discussion is from the observer H. He saw that the speed of the train would not be $\mathrm{v}$ definitely, so he divided the speed of the train into two speeds, one is the instant speed corresponding to the observer $\mathrm{H}$, namely $H=v \cos \angle \theta \beta$, the time error value in front of the car is $+\Delta l=-l \cos \angle \theta \beta$, the length light error value is $+\Delta l=-l \cos \angle \theta \beta$, it is $-\Delta t=t \cos (\pi-\angle \theta) \beta,-\Delta l=l \cos (\pi-\angle \theta) \beta$ behind the train. The light error values in the front position and the rear position is a total of $(+\Delta t)+(-\Delta t)=0$ and $(+\Delta l)+(-\Delta l)=0$. Therefore, the total observing length of the front and the rear of the train by the observer $\mathrm{H}$ is not changed. The observer only observes the virtual image position, then the real image position of the object must be supplemented by relativity.

When $\angle \theta=0$, the speed of observer A to observe the train can be obtained, namely $A=v \cos 0=v$, light error is $+\Delta t=t \beta,+\Delta l=-l \beta$. When $\angle \theta=\pi$, the speed of the observer B to observe the train can be obtained, namely $B=v \cos \pi=-v$, the speed direction is contrary to point $\mathrm{A}$, light error is $-\Delta t=-t \beta,-\Delta l=l \beta$. Observer A can only observe the trail of the train, but not the head of the train. The observed virtual image is longer than the object, and the time slows down. On the contrary, the virtual image of B is shorter than the object and the time is faster. Their light errors are added to obtain the object length of the virtual image, which is the same as the actual train.

Therefore, the follows is obtained according to the above relationships.

When $\beta>0$, the object is moving away from the observer, and the virtual image is moving away from the real image at the speed $1+|\beta|$.

When $\beta<0$, the object is approaching the observer; the virtual image approaches the real image at the speed $1+|\beta|$

When $\beta=0$, the object and the observer's positions coincide; the virtual and real images coincide.

However, movement inevitably produces light error. Because the information source of light is accepted currently. Both electromagnetic wave and optical instruments are receiving speed of the light speed $\mathrm{C}$. The real information of the object can be obtained through Newtonian mechanics. However, it only obtain light virtual information, and the real information can be obtained through describing the observer's theory of relativity for correction. They are completely unified, which complement each other regardless of low speed and high speed.

The discovery of human beings to light error information is actually earlier in the theory of relativity. 
$\beta t=\Delta T$ can be obtained through the above examples. $\Delta T$ refers to total error value. It seems strange, which can be understood through dismantling. Then, $\frac{v^{*} t}{c}=\Delta T \cdot \mathrm{v}$ is changed into $\mathrm{c}, \mathrm{t}$ is changed into the time of 1 year, namely $\frac{\text { Distance of travling for one year at light speed }}{\text { Lightspeed }}=1$ year. Therefore, the commonly used unit light year in astronomy can be obtained, namely a common astronomy phenomenon. The observed planet with the distance of 1 light year is just the image of the planet one year ago. In addition, light error can be used for explaining Yang Zhenning's parity nonconservation principle by optical error, so it didn’t find anything.

Then, the question of the first picture can be answered very simply as shown in Figure 6.

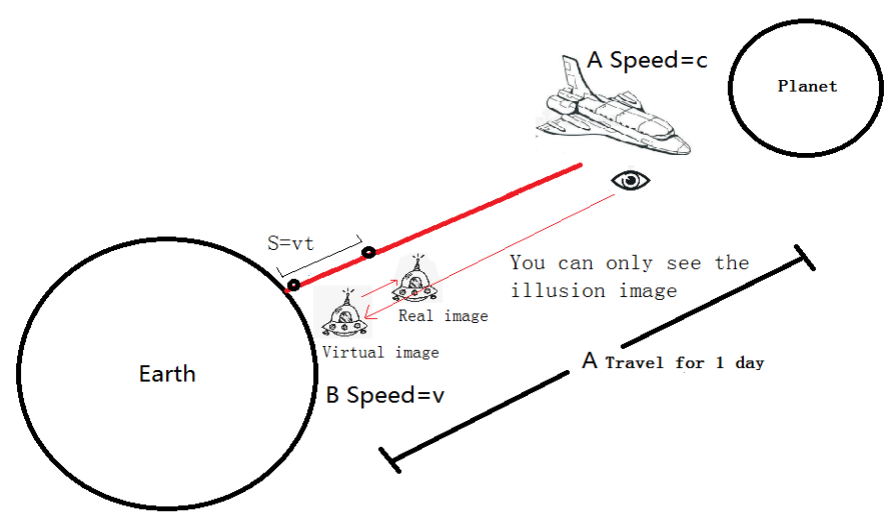

Figure 6. Final trajectory figure of planet and earth.

The answers can be summarized to the above two questions as follows accordingly.

Answer to question 1: the monkey spacecraft and the light spacecraft are moved at the same time, which travel for a day at the speed $\mathrm{v}$, and the distance is $\mathrm{S}$.

Answer to question 2: because the speed is $\mathrm{C}$, the light image transmission rate is also $\mathrm{C}$, the speed of receiving information is 0 , then the monkey spacecraft and earth are almost moveless from the spacecraft according to the speed of the spacecraft, namely the time is stopped. After the spacecraft reaches the planet, the movement of the earth and the monkey spacecraft are started, namely virtual image one day ago. The monkey spacecraft is moved to the real image at the speed faster than the original speed by $\beta$ times. When the virtual image and real image overlap, the spacecraft reaches the planet and is located at the same position as the spacecraft. This is the image of both parties to observe the earth one day ago ${ }^{[8]}$. The light error time with the earth is one day. Meanwhile, if the light aircraft flies to the earth, light information of twice speed can be observed. The catching real images can be played aiming at the earth virtual image at twice speed, thereby supplementing the time of the day.

Therefore, all the discussion results are combined. It can be concluded that there is no contradiction between relativity and Newtonian mechanics, and the essence of the relativity lies in the mass energy equation. The theoretical results described above are only used as reference.

Code 6: 1644505015261

Code 7: 29789564915261

Code 8: 886524815261

Code 9: 19194744756666

Code 10: 11011004615131

\section{References}

1. Jin Y. Supplement to a problem related to relativity. Physics Teaching 2008; (11): F0003-F0003.

2. Shu M. Addendum to mass energy formula and special relativity (in Chinese). Journal of Hubei Agricultural University 1985; (6): 43-49. doi: CNKI:SUN:HBNX.0.1985-06-004.

3. Zhou W. Feasibility study of the unity theory of quantum mechanics, classical mechanics, relativistic mechanics 
(14): Supplement to the new quantum mechanics (1) (in Chinese). Public Communication of Science \& Technology 2019; 11(5): 174-176. doi: 10.16607/j.cnki.1674-6708.2019.05.081.

4. Yin R. The experimental proof for rotating theory of relativity. Journal of System Simulation 1999; 11(4): 302-306. doi: 10.3969/j.issn.1004-731X.1999.04.022.

5. Dong H. Relativity of litigation object: From the perspective of procedural guarantee theory. Contemporary Law, 33(02): 130-139.

6. Guo Y, Shen H. History of physics. Tsinghua University Press (2nd Edition); August 2005.

7. Ma W. Physics. Higher Education Press (5th Edition); April 2006.

8. Zhou W. Continued on several issues related to associated energy conservation, i.e. energy conservation. Public Communication of Science \& Technology 2014; (3): 111. 